Research Paper:

\title{
Estimation of Median Nerve Axonal Degeneration without Needle Electromyography
}

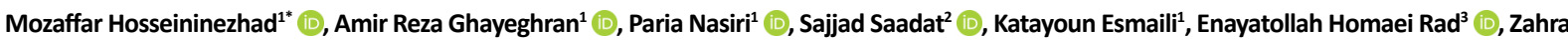
Gholipour Soleimani ${ }^{4}$

1. Department of Neurology, Guilan Road Trauma Research Center, Guilan University of Medical Sciences, Rasht, Iran

2. Neuroscience Research Center, Guilan University of Medical Sciences, Rasht, Iran.

3. Research Assistant Professor of Health Economics, Guilan Road Trauma Research Center, Guilan University of Medical Sciences, Rasht, Iran.

4. Department of Nursing, Neuroscience Research Center, Guilan University of Medical Sciences, Rasht, Iran.

\begin{tabular}{|c|c|}
\hline $\begin{array}{l}\text { Use your device to scan } \\
\text { and read the article online }\end{array}$ & Citration: Hosseininezhad M, Ghayeghran AR, Nasiri P, Saadat S, Esmaili K, Homaei Rad E, et al. Estimation of Median Nerve \\
\hline 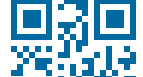 & $\begin{array}{l}\text { Axonal Degeneration Without Needle Electromyography. Iran J Neurosurg. 2021; 7(1):23-30. http://dx.doi.org/10.32598/ir- } \\
\text { jns.7.1.4 }\end{array}$ \\
\hline 口atid & doli http://dx.doi.org/10.32598/irjns.7.1.4 \\
\hline
\end{tabular}

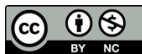

Article info:

Received: 1 Sep 2020

Accepted: 1 Nov 2020

Available Online: 01 Jan 2021

Keywords:

Median nerve, Electromyography, Carpal tunnel syndrome

\section{A B S T RACT}

Background and Aim: The present study aimed to use the median nerve Compound Muscle Action Potential (CMAP) amplitude by stimulation at the palm instead of Abductor Pollicis Brevis (APB) needle Electromyography (EMG) for determining axonal loss in patients with Carpal Tunnel Syndrome (CTS).

Methods and Materials/Patients: This study was performed on 180 patients with CTS referred to the Electrodiagnostic (EDX) Center, Poursina Hospital, Guilan Province, Iran, in 2018-19. In this study, the APB needle EMG diagnostic test was used as the gold standard, and median nerve CMAP amplitude with stimulation at the palm and wrist were used to compare the two nerve stimulation tests.

Results: All of the cases with abnormal amplitude loss detected by median nerve stimulation at the palm also had an axonal loss in the needle EMG of APB. So this test could be a good indicator of axonal loss if there is an abnormality (sensitivity: 73\%, specificity: 100\%). The results with wrist stimulation were not as accurate as of the palm stimulation, and some cases with decreased CMAP amplitude of median nerve had normal needle EMG of APB muscle (sensitivity: $86.6 \%$, specificity: 94.9\%).

Conclusion: In cases with CTS, the abnormally decreased amplitude of the median nerve detected by stimulation at the palm could be a good indicator of axonal loss.

* Corresponding Author:

Mozaffar Hosseininezhad, MD.

Address: Department of Neurology, Guilan Road Trauma Research Center, Guilan University of Medical Sciences, Rasht, Iran Tel: +98 (13) 33322444

E-mail: hosseininezhadm@gmail.com 


\section{Highlights}

- All patients with carpal tunnel syndrome with axonal degeneration in needle EMG of abductor pollicis brevis (APB) muscle showed abnormal amplitude loss during median nerve stimulation at the palm.

- The sensitivity and specificity of recording median nerve amplitude with stimulation at palm were $73 \%$ and $100 \%$, respectively.

- The sensitivity and specificity of recording median nerve amplitude with stimulation at the wrist were $86.6 \%$ and $94.9 \%$, respectively.

\section{Plain Language Summary}

In this project, we intend to investigate whether median nerve stimulation at the palm and wrist could accurately detect axonal loss in patients with Carpal Tunnel Syndrome (CTS) similar to Electromyography (EMG) on the Abductor Pollicis Brevis (APB) muscle. In this regard, we studied 180 patients with CTS referred to the Electrodiagnostic (EDX) Center at Poursina Hospital, Guilan Province, Iran, between 2018 and 2019. We determined the signs of axonal loss in the patients using two tests, including median nerve stimulation at palm and wrist. We compared the results of these tests with EMG outputs for APB as a gold standard to clarify the sensitivity and specificity of two tests to diagnose axonal loss in CTS patients. Our findings showed that an abnormal amplitude loss during median nerve stimulation at palm like the axonal loss diagnosed by EMG of APB was observed in all patients. We estimated the sensitivity of $73 \%$ and $86 \%$ and the specificity of $100 \%$ and $94.9 \%$ for median nerve stimulation at palm and wrist, respectively. As a result, we found an abnormal amplitude loss during median nerve stimulation at palm that could be a good indicator for diagnosing axonal loss in CTS patients. This test might be used instead of the EMG of APB.

\section{Introduction}

arpal Tunnel Syndrome (CTS) is common in the general population and more prevalent in women. The classic symptoms of CTS are numbness and paranesthesia in the first three fingers of the hand, more at night [1]. The signs include sensory loss along the lateral aspect of the hand, motor weakness and wasting of Abductor Pollicis Brevis (APB) muscle, and eliciting Tinel's and Phalen's signs at the wrist. Electrophysiological findings, including Nerve Conduction Studies (NCS) and needle Electromyography (EMG), are quite valuable for diagnosis and grading CTS $[2,3]$. In general, NCS is more valuable than needle EMG in the diagnosis of CTS because the underlying pathophysiology is mainly focal demyelination. If the sensory and motor distal latency in the wrist is prolonged, it strongly suggests CTS [4].

The Compound Muscle Action Potential (CMAP) amplitude and Sensory Nerve Action Potential (SNAP) amplitude may reduce due to secondary axonal degeneration or conduction block [5]. The only way to differentiate the two is to stimulate the median nerve in the palm and compare its amplitude with the wrist stimulation. If the amplitude ratio of the palm to the wrist is greater than
1.6 for sensory and more than 1.2 for the motor, it will be a sign of conduction block [6, 7]. However, the needle EMG is often helpful in further characterizing the neuropathic insult in CTS, especially when the CMAP amplitude is reduced because this condition can be a consequence of either distal demyelination or axonal degeneration. In addition, needle examination is essential for identifying proximal lesions such as cervical radiculopathy or more proximal median nerve entrapment.

Recommended muscles for needle EMG include APB and at least two muscles innervated by $\mathrm{C} 6$ and $\mathrm{C} 7$ roots such as flexor carpi radialis and triceps to exclude cervical radiculopathy. If needle EMG of APB muscle is abnormal, at least one proximal muscle with median innervation and two muscles that innervate the roots of C8 and T1 should also undergo EMG. Needle EMG of APB muscle is often painful and unbearable for some patients, and it is best not to be the first but the last muscle for this test [8].

Needle EMG helps determine the nature of nerve injury by demonstrating evidence of denervation in muscle, especially when CMAP amplitude wave is reduced, which may be due to conduction block or axonal loss. On the other hand, axonal degeneration in CTS is 
uncommon, and studies suggest that findings such as fibrillation and Positive Sharp Wave (PSW) cannot be used as a measure of the severity of motor axon injury. Also, using the needle EMG in CTS patients has been considered unreliable and controversial [9].

Because of the painfulness of the needle EMG, some patients cannot tolerate this part of the test, and sometimes the patient leaves the test with an unfavorable memory. Arabadzhiev et al. [10] studied the factors affecting pain during EMG. Their study revealed that the APB muscle suffered the most severe pain. In another study conducted by Marva Mohamed El-Emary and Hasan [11] in Vienna, Austria, CMAP amplitude was reported as the strongest predictor of axonal loss, but it was noted that needle EMG is still required in some cases of CTS. However, this study did not indicate the site of the nerve stimulation.

As we already mentioned, the needle EMG is a painful procedure, especially in APB muscle. However, it is possible to diagnose CTS and axonal loss with NCS findings without using a needle for this muscle. Thus, the replacement of needle EMG with this method will increase the patient's comfort and convenience.

The Electrodiagnostic (EDX) Center at Poursina Hospital is the only university center in Guilan Province equipped with all the facilities to perform electrodiagnostic procedures and is one of the important referral centers for examining CTS due to the cooperation of neurologists, orthopedists, and neurosurgeons in this hospital. Therefore, we decided to examine the use of median nerve wave amplitude criterion by palm stimulation instead of APB needle EMG as an index of axonal loss in this center.

\section{Methods and Materials/Patients}

This cross-sectional descriptive study evaluated CTS diagnostic tests. This research has been registered with the code number IR.GUMS.REC.1397.243 in the National Ethics Committee of Guilan University of Medical Sciences. In this study, we included 180 patients with moderate to severe CTS referred to the EDX Center at Poursina Hospital in 2018-19. The APB needle EMG diagnostic test was used as the gold standard to compare the two tests of median nerve stimulation at the palm and wrist. The exclusion criteria included evidence of peripheral nerve injury other than CTS; radiculopathy at the root of $\mathrm{C} 8$ and $\mathrm{T} 1$; having diseases that inhibit EMG, such as blood and infectious disorders in needle site; having local problems at the site of nerve stimulation in the wrist or palm, and intolerance and dissatisfaction of the patient to continue the EMG process. To calculate the sensitivity and specificity, a sample size formula related to this type of study was used. In this formula, the "sens" is the sensitivity of the test in previous studies, " $d$ " is the acceptable error, which here is equal to 0.1 , and "prev" is the probability of occurrence used for determining the sensitivity of the test. According to Fleming et al. study, the sensitivity of the palm method was 0.47 , and the probability of occurrence was $40 \%$ [12]. Therefore, the sample size in this study was estimated to be 168 cases, which was increased to 180 .

All patients referred for EMG in CTS were invited to perform the test. Then, all routine diagnostic procedures, including sensory and motor recordings and needle EMG examination, were performed. In cases in which CTS was moderate or severe, median stimulation in the palm was conducted in addition to doing the standard measures, and the resulting response was recorded. Then, the motor amplitude resulting from this stimulation was compared with that from the wrist stimulation. Patients were categorized into three groups according to the results of median nerve motor response with stimulation at the palm and wrist.

The cases with normal limit motor responses in palm and wrist stimulation without conduction block were categorized in the first group. Cases where the CMAP amplitude is reduced by wrist stimulation and is normal by palm stimulation were put in the second group, and cases where the amplitude of both are reduced and abnormal were put in the third group. Then, the considered muscles, including APB, were needled. Findings of APB needle EMG were examined for the presence or absence of axonal loss in three separate groups.

A checklist, including age, gender, medical history, EMG, and NCS results, was completed. One researcher performed all tests, so there was no problem with the validity and reliability of the two tests. Then, the sensitivity, specificity, and positive and negative predictive value of the median nerve stimulation tests by the palm and wrist were calculated using the following formulas. In this diagnostic study, false positives, false negatives, true positives, and true negatives are determined. The sensitivity index is equal to the ratio of true positives to the sum of true positives and false negatives. The specificity index is the ratio of true negatives to the sum of true negatives and false positives. The description of positive and negative predictive values are as follows: 
Positive predictive value: The ratio of true positives to the sum of true positives and false positives, Negative predictive value: The ratio of true negatives to the sum of true negatives and false negatives.

In addition to calculating the sensitivity and specificity of the diagnostic test using regression patterns, the effect of the variables that impact false negatives or false positives was investigated. The dependent variable of this pattern was false test results, and independent variables included age, gender, medical history, among others. All statistical information was collected by Excel 2016 and analyzed by Stata 16 software.

\section{Results}

In the present study, 180 patients participated, of whom 146 people (91.11\%) were women. The mean age of the patients was 49.33 years (age range: $26-81$ years). A total of 120 participants (66.67\%) were righthanded. As shown in Table 1, examining median nerve amplitude by palm stimulation showed that 52 patients had a decrease in amplitude, indicating an axonal loss. In all of them, the APB needle EMG showed the same result. Also, 31 out of 128 patients with normal motor amplitude in the median nerve showed an axonal loss in the needle EMG of APB muscle.

Table 2 presents the sensitivity, specificity, positive and negative predictive values of the diagnostic test for stimulating the median nerve in the palm compared to needle EMG. With the probability of occurrence of $53 \%$, sensitivity was reported at $73 \%$, specificity $100 \%$, the positive predictive value of $100 \%$, and the negative predictive value of $82.9 \%$. Also, after examining median nerve amplitude by wrist stimulation, it was found that 65 out of 75 patients with decreased motor amplitude of median nerve in the wrist showed an axonal loss in needle EMG. Furthermore, 18 out of 105 patients with normal motor amplitude of median nerve showed axonal loss in APB needle EMG.
Table 3 presents the sensitivity, specificity, the positive and negative predictive values of the median nerve with wrist stimulation diagnostic test compared with APB needle EMG with the probability occurrence of $53 \%$. The sensitivity of the test is $86.6 \%$, specificity $94.9 \%$, a positive predictive value of $93.4 \%$, and a negative predictive value of $89.5 \%$.

Table 4 presents the effect of age and gender on falsepositive cases of diagnostic test on median nerve stimulation of the wrist compared to APB needle EMG. The results expressed a statistically significant relationship between the age of people and false positives $(P=0.05)$ so the older the age, the lower the number of false positives given to the negative correlation coefficient of -0.16 .

\section{Discussion}

This study was performed to evaluate the predictive value of median nerve motor amplitude in palm stimulation for detecting an axonal loss in patients with CTS. There are many diagnostic procedures for examining and diagnosing CTS in EDX review [13]. While there are studies that suggest surgeons perform surgery without electrodiagnosis in patients with CTS symptoms, most surgeons agree that diagnostic tests should confirm CTS because the outcome of the surgery is better when the nerve is released before the significant destruction of the axon [14]. Although there are standards for testing this syndrome, there are still controversies about the available tests. APB needle EMG is routinely performed to diagnose axonal loss of the median nerve, and this procedure is usually painful, sometimes causing patients to refuse to test [13]. This study aimed to determine if it is possible to use the CMAP amplitude of the median nerve instead of the needle EMG of APB muscle to determine the axonal loss of the median nerve.

This study found that all cases with abnormally decreased CMAP amplitude of the median nerve with stimulation in the palm showed evidence of axonal loss in the needle EMG of ABP muscle as well, so this test

Table 1. Determining the CTS using the median nerve amplitude method and needle EMG

\begin{tabular}{cccc}
\hline $\begin{array}{c}\text { Median Motor Amplitude in Palm } \\
\text { Stimulation }\end{array}$ & $\begin{array}{c}\text { Axonal Loss in Abductor Pollicis } \\
\text { Brevis Needle EMG }\end{array}$ & $\begin{array}{c}\text { Normal Abductor Pollicis Brevis } \\
\text { Needle EMG }\end{array}$ & Total \\
\hline Normal & 31 & 97 & 128 \\
Abnormal & 52 & 0 & 52 \\
Total & 83 & 97 & 180 \\
\hline
\end{tabular}


Table 2. Predictive values of the diagnostic test of median nerve stimulation in the palm and needle EMG

\begin{tabular}{cc}
\hline Probability of Occurrence & $\mathbf{5 3 \%}$ \\
\hline Sensitivity & $73 \%$ \\
Specificity & $100 \%$ \\
Positive predictive value & $100 \%$ \\
Negative predictive value & $82.9 \%$ \\
\hline &
\end{tabular}

could be an excellent way to predict axonal loss. Elmary et al. assessed the value of the needle EMG compared to NCV (Nerve Conduction Velocity) findings [11]. They showed that among the parameters examined in NCV, CMAP could be the strongest predictor of spontaneous activity in EMG and reduces the need for the needle EMG in some patients [11]. Another study evaluated the association between NCV and EMG tests, where CMAP amplitude can be used as a strong predictor instead of needle EMG [15]. The results of Chang et al. study are also consistent with the present study's findings [15].

Kogelschatz et al. [16] reviewed the role of EMG in CTS. Their results indicated that if there was a high probability of detecting CTS in NCV, it is not necessary to perform EMG [16]. As the EMG is used to diagnose CTS and determine its severity to help the patient and physician choose the best treatment, it is possible to use this method in certain cases to discontinue doing APB needle EMG. Also, nerve injury in CTS is mainly in the form of demyelination in the wrist; the conduction block in the wrist can cause amplitude to drop and falsely predict axonal loss. In this study, besides the wrist, the palm was stimulated, which was after the nerve entrapment area, and no change was expected in the distal parts of the nerve by demyelinating damage in the wrist. If there is a drop in amplitude in the test of this part, it can be an excellent criterion for axonal loss.
Rubin et al. reported that in patients with CTS diagnosis without the involvement of the motor part of the median nerve, the likelihood of occurrence of an abnormal EMG in APB muscle is very low, and this test is not required [17]. This study suggests that EMG is required in patients with prolonged distal latency but whose CMAP amplitude is normal because EMG may help diagnose axonal degeneration in this condition.

In our study, in some cases with normal responses of median nerve CMAP with nerve stimulation at the wrist and palm, there was the abnormal needle EMG of APB muscle indicating axonal loss, and accordingly, needle EMG is recommended in these cases. Indeed, the diagnostic sensitivity of this test was not as helpful as the specificity that can be due to the following reasons.

Firstly, the motor amplitude of the median nerve less than $4 \mathrm{mv}$ is considered abnormal, so there are cases where the amplitude has not decreased to $4 \mathrm{mv}$, but there is axonal loss. In these cases, it can be helpful if the patient has had a previous test and its results are compared with the new CMAP amplitude test result. However, if this opportunity does not exist, it is not possible to compare, and performing this test alone does not seem to be sufficient to diagnose axonal loss. Secondly, a slight axonal loss may be detected on the needle EMG that does not alter the CMAP amplitude in some cases.

Table 3. Predictive values of the diagnostic test of median nerve stimulation in the wrist and APB needle EMG

\begin{tabular}{cc}
\hline Probability of Occurrence & $\mathbf{5 3 \%}$ \\
\hline Sensitivity & $86.6 \%$ \\
Specificity & $94.9 \%$ \\
Positive predictive value & $93.4 \%$ \\
Negative predictive value & $89.5 \%$ \\
\hline
\end{tabular}


Table 4. Effect of age and gender on diagnosis of medinan axonal degeneration with CMAP and needle examination

\begin{tabular}{cccccc}
\hline False-positive Cases & Correlation Coefficient & Standard Error & P Coefficient & Minimum & Maximum \\
\hline Gender & -0.16 & 0.74 & 0.82 & -1.62 & -0.11 \\
Age & -0.06 & 0.02 & 0.05 & -0.00 \\
\hline
\end{tabular}

The effect of age, sex, and history of the disease on false-positive results showed a significant relationship between age and false positives so that the false-positive results decrease with increasing age according to the negative correlation coefficient of -0.16 . Given that the cause of being false positive in wrist stimulation is the conduction block, this finding suggests that the decrease in amplitude in the wrist stimulation at older ages is more likely due to the axonal loss and less due to conduction block. This condition increases true positives cases. This finding is consistent with the results of the Gnatz's study [18]. They found that patients with more severe CTS were in older age groups. Also, Werner and Andary demonstrated that people with abnormal results in the EMG test were older, which may be due to the higher possibility of axonal loss in CTS in older people compared to other age groups [19]. The present result is consistent with the study's result of El-Emary and Hassan [11]. Other studies have shown that most patients (80\%) were women between 19 and 75 [5].

Needle EMG must be used to differentiate other diagnostic cases, especially cervical radiculopathy. Zuniga et al. examined the need for EMG in CTS, and found that $19 \%$ of patients who participated in the study had cervical radiculopathy and CTS simultaneously [20].

\section{Conclusion}

According to the study findings and its compatibility with other studies, it can be inferred that in patients with moderate and severe CTS, if the motor amplitude of the median nerve with stimulation in the wrist is normal, there is no need to stimulate the nerve in the palm. However, given that there may be a possibility of minor axonal loss in the needle EMG of APB muscle in these cases if this finding changes the treatment process (surgical indication), the APB needle EMG should also be performed.

If the motor amplitude of the median nerve with stimulation in the wrist is low, it should be tested by stimulating the palm. If there is a drop in amplitude in the stimulation of the palm, it strongly suggests axonal loss, and there is no need to do APB needle EMG for confirming axonal loss. However, if the amplitude with stimulation of the palm is normal, a conduction block is considered, and the need for needle EMG is similar to the cases in which the amplitude of the wrist was normal.

Importantly, determining the median CMAP changes can predict the existence of axonal loss and also its severity, but it could not predict the time of nerve injury. For this purpose, only medical history and needle EMG can be helpful. Although predicting the time of developing axonal loss may not alter the treatment process of these patients, this is one of the main drawbacks of this test. Given that the decrease in median CMAP amplitude with stimulation of the palm is a good predictor of axonal loss in APB muscle, the needle EMG to diagnose axonal loss can be avoided in certain cases, if necessary. For further study, it is suggested that the needle EMG changes in the APB muscle be identified separately in different amplitudes, and if it is helpful, a specific amplitude can be determined as the specific criterion for avoiding the needle EMG.

This study had some limitations, such as clients' refusing to do the needle EMG because it was painful. To reduce this limitation, an attempt was made to explain the importance of the test for diagnosis to the patient, and the needle EMG was performed on APB muscle near the end of the test, and finally, if the patient did not continue the test, s/he was excluded from the study and replaced with another patient.

\section{Ethical Considerations}

\section{Compliance with ethical guidelines}

Patients signed a written informed consent to participate in the study. This study was conducted following the principles of the Declaration of Helsinki. The Ethics Committee of Guilan University of Medical Sciences approved the study protocol. 
Funding

This research did not receive any grant from funding agencies in the public, commercial, or non-profit sectors.

\section{Authors' contributions}

All authors contributed equally to performing the project and preparing the manuscript.

\section{Conflict of interest}

The authors declared no conflict of interest.

\section{Acknowledgments}

We would like to thank all study participants and the Deputy of Technology and Treatment of Guilan University of Medical Sciences.

\section{References}

[1] Aboonq MS. Al-hijamah (wet cupping therapy of prophetic medicine) as a novel alternative to surgery for carpal tunnel syndrome. Neurosciences. 2019; 24(2):137-41. [DOI:10.17712/ nsj.2019.2.20180036] [PMID]

[2] Gillig JD, White SD, Rachel JN. Acute carpal tunnel syndrome A review of current literature. Orthopedic Clinics of North America. 2016; 47(3):599-607. [DOI:10.1016/j.ocl.2016.03.005] [PMID]

[3] MacDermid JC, Doherty T. Clinical and electrodiagnostic testing of carpal tunnel syndrome: A narrative review. Journal of Orthopaedic and Sports Physical Therapy. 2004; 34(10):565-88. [DOI:10.2519/jospt.2004.34.10.565] [PMID]

[4] Preston DC, Shapiro BE. Needle electromyography fundamentals, normal and abnormal patterns. Neurologic Clinics. 2002; 20(2):361-96. [DOI:10.1016/S0733-8619(01)00005-6] [PMID]

[5] Shapiro LJ, Jungers WL. Electromyography of back muscles during quadrupedal and bipedal walking in primates. American Journal of Physical Anthropology. 1994; 93(4):491-504. [DOI:10.1002/ajpa.1330930408] [PMID]

[6] Arab AA, Elmaghrabi MM, Eltantawy MH. Carpal tunnel syndrome: Evaluation of its provocative clinical tests. Egyptian Journal of Neurosurgery. 2018; 33(1):1-12. [DOI:10.1186/s41984018-0014-7]

[7] Baričić M, Šantić V, Legović D, Jotanović Z, Matejččć N, Barković MM. Carpal tunnel syndrome. Medicina Fluminensis. 2019; 55(1):4-15. [DOI:10.21860/medflum2019_216313]

[8] Kurver A, Smolders J, Verhagen WIM, Meulstee J, Nijhuis FAP The diagnostic sensitivity for ulnar neuropathy at the elbow is not increased by addition of needle EMG of ADM and FDI when nerve conduction studies are normal. Front Neurol. 2019; 10:196 [DOI:10.3389/fneur.2019.00196] [PMID] [PMCID]
[9] Sonoo M, Menkes DL, Bland JDP, Burke D. Nerve conduction studies and EMG in carpal tunnel syndrome: Do they add value? Clinical Neurophysiology Practice. 2018; 3:78-88. [DOI:10.1016/j.cnp.2018.02.005] [PMID] [PMCID]

[10] Arabadzhiev TI, Dimitrov GV, Dimitrov AG, Chakarov VE, Dimitrova NA. Factors affecting the turns analysis of the interference EMG signal. Biomedical Signal Processing and Control. 2008; 3(2):145-53. https://www.researchgate.net/ publication/237898326_Factors_affecting_the_turns_analysis_of_the_interference_EMG_signal

[11] El-Emary WS, Hassan MM. Needle electromyography in carpal tunnel syndrome: Is it valuable or predictable? Egyptian Rheumatology and Rehabilitation. 2016; 43(1):41-6. https:/ / erar.springeropen.com/articles/10.4103/1110-161X.177426

[12] Fleming N, Donne B, Fletcher D. Effect of kayak ergometer elastic tension on upper limb EMG activity and 3D kinematics. Journal of Sports Science \& Medicine. 2012; 11(3):430-7 [PMID] [PMCID]

[13] Goyal V, Bhatia M, Padma MV, Jain S, Maheshwari MC Electrophysiological evaluation of 140 hands with carpal tunnel syndrome. The Journal of the Association of Physicians of India. 2001; 49:1070-3. [PMID]

[14] Fowler JR. Nerve conduction studies for carpal tunnel syndrome: Gold standard or unnecessary evil? Orthopedics. 2017 40(3):141-2. [DOI:10.3928/01477447-20170419-01] [PMID]

[15] Chang CW, Lee WJ, Liao YC, Chang MH. Which nerve conduction parameters can predict spontaneous electromyographic activity in carpal tunnel syndrome? Clinical Neurophysiology. 2013; 124(11):2264-8. [DOI:10.1016/j. clinph.2012.03.019] [PMID]

[16] Kogelschatz C, Laughlin R. The role of needle electromyography in the evaluation of carpal tunnel syndrome (P2. 460). Neurology. 2018; 90 (Suppl 15):1-4. https://n.neurology.org/ content/90/15_Supplement/P2.460.abstract

[17] Rubin DI, Dimberg EL. Needle EMG of thenar muscles in less severe carpal tunnel syndrome. Journal of Clinical Neurophysiology. 2018; 35(6):481-4. [DOI:10.1097/ WNP.0000000000000519] [PMID]

[18] Gnatz SM. The role of needle electromyography in the evaluation of patients with carpal tunnel syndrome: Needle EMG is important. Muscle and Nerve. 1999; 22(2):282-3. [DOI:10.1002/ (SICI)1097-4598(199902)22:2<282::AID-MUS19>3.0.CO;2-N]

[19] Werner RA, Andary M. Electrodiagnostic evaluation of carpal tunnel syndrome. Muscle and Nerve. 2011; 44(4):597-607. [DOI:10.1002/mus.22208] [PMID]

[20] Zuniga LA, Ross M. T34. Diagnosing patients with carpal tunnel syndrome: Do you need needle EMG? Clinical Neurophysiology. 2018; 129(Supp 1):e14. [DOI:10.1016/j. clinph.2018.04.035] 
This Page Intentionally Left Blank 